\title{
A Multicenter Pragmatic Interrupted Time Series Analysis of Chlorhexidine Gluconate Bathing in Community Hospital Intensive Care Units
}

\author{
Kristen V. Dicks, MD, MPH ${ }^{1,2}$, Eric Lofgren, MSPH, $\mathrm{PhD}^{3}$, Sarah S. Lewis, MD, MPH ${ }^{1,2}$, \\ Rebekah W. Moehring, MD, MPH ${ }^{1,2,4}$, Daniel J. Sexton, MD, FIDSA ${ }^{1,2}$, and Deverick J. \\ Anderson, MD, MPH ${ }^{1,2}$ \\ ${ }^{1}$ Department of Medicine, Division of Infectious Diseases, Duke University Medical Center, \\ Durham, North Carolina \\ 2Duke Infection Control Outreach Network, Durham, North Carolina \\ ${ }^{3}$ Network Dynamics and Simulation Science Lab, Virginia Bioinformatics Institute, Virginia Tech, \\ Blacksburg, Virginia \\ ${ }^{4}$ Durham Veterans Affairs Medical Center, Durham, North Carolina
}

\section{Abstract}

Objective-To determine whether daily chlorhexidine gluconate (CHG) bathing of intensive care unit (ICU) patients leads to a decrease in hospital-acquired infections (HAIs), particularly infections caused by methicillin-resistant Staphylococcus aureus (MRSA) and vancomycinresistant Enterococcus (VRE).

Design-Interrupted time series analysis.

Setting-The study included 33 community hospitals participating in the Duke Infection Control Outreach Network from January 2008 through December 2013.

Participants-All ICU patients at study hospitals during the study period.

Methods-Of the 33 hospitals, 17 hospitals implemented CHG bathing during the study period, and 16 hospitals that did not perform CHG bathing served as controls. Primary pre-specified outcomes included ICU central-line-associated bloodstream infections (CLABSIs), primary bloodstream infections (BSI), ventilator-associated pneumonia (VAP), and catheter-associated urinary tract infections (CAUTIs). MRSA and VRE HAIs were also evaluated.

Results-Chlorhexidine gluconate (CHG) bathing was associated with a significant downward trend in incidence rates of ICU CLABSI (incidence rate ratio [IRR], 0.96; 95\% confidence interval [CI], 0.93-0.99), ICU primary BSI (IRR, 0.96; 95\% CI, 0.94-0.99), VRE CLABSIs (IRR, 0.97; 95\% CI, 0.97-0.98), and all combined VRE infections (IRR, 0.96; 95\% CI, 0.93-1.00). No

Address correspondence to Kristen V. Dicks, MD, MPH, PO Box 102359, Department of Medicine, Division of Infectious Diseases, Duke University Medical Center, Durham, NC 27710 (Kristen.dicks@duke.edu).

Previous Presentation. An abstract containing preliminary data was presented at IDWeek, October 8, 2015, San Diego, California. 
significant trend in MRSA infection incidence rates was identified prior to or following the implementation of CHG bathing.

Conclusions-In this multicenter, real-world analysis of the impact of CHG bathing, hospitals that implemented CHG bathing attained a decrease in ICU CLABSIs, ICU primary BSIs, and VRE CLABSIs. CHG bathing did not affect rates of specific or overall infections due to MRSA. Our findings support daily CHG bathing of ICU patients.

Hospital-acquired infections (HAIs) are harmful and expensive complications of healthcare. In 2011, 722,000 HAIs occurred in United States hospitals and were associated with 75,000 deaths. ${ }^{1}$ The total annual costs for HAIs approach $\$ 10$ billion in the United States. ${ }^{2}$ HAIs due to multidrug-resistant organisms (MDROs), such as methicillin-resistant Staphylococcus aureus (MRSA) and vancomycin-resistant Enterococcus (VRE), are associated with higher mortality rates and costs than HAIs due to non-MDRO HAIs. ${ }^{3-7}$

Hospitals currently allocate a substantial amount of effort and resources toward monitoring and preventing HAIs. For example, most hospitals use contact precautions for patients who are colonized with these organisms and also use active surveillance methods to detect colonization. Patient bathing with chlorhexidine gluconate $(\mathrm{CHG})$ has been widely implemented as an additional strategy to combat HAIs. CHG has antimicrobial activity against Gram-positive and Gram-negative bacteria and decreases bacterial colony counts on the surface of the skin. ${ }^{8}$ As a result, $\mathrm{CHG}$ has been used to lower rates of blood culture contamination, ${ }^{9}$ environmental contamination, ${ }^{10}$ and surgical site infection. ${ }^{11}$

The impact of CHG bathing on HAI prevention in intensive care units (ICUs) has been examined in quasi-experimental ${ }^{9,12-16}$ and cluster-randomized studies with and without crossover, ${ }^{17-20}$ and by meta-analyses, ${ }^{21,22}$ with varying endpoints and results. However, to our knowledge, no previous studies have examined the impact of CHG bathing on HAIs in a multi-center, observational manner. Thus, the effectiveness of $\mathrm{CHG}$ bathing in a real-world, type 3 translational research setting is unknown.

Our goal was to estimate the effect of CHG bathing on the incidence of ICU-acquired HAIs, including MRSA and VRE infections, using time-trended surveillance data from a large network of community hospitals.

\section{Methods}

\section{Study Setting and Design}

We conducted an interrupted time series analysis of the effect of using CHG bathing on 13 potential infection-related outcomes of interest in hospitals participating in the Duke Infection Control Outreach Network (DICON). DICON is a network of more than 40 community hospitals in 5 states throughout the southeastern United States that has been described previously. ${ }^{23}$ DICON provides infection control resources and services from liaison infection preventionists and physician epidemiologists. Infection preventionists at each hospital collect surveillance data using a standardized database employing National Healthcare Safety Network (NHSN) definitions. Liaison infection preventionists validate a subset of surveillance data at each hospital each month. Decisions to use CHG bathing were 
made by each hospital independently and were not controlled by researchers but were relayed to researchers by local infection control teams. All ICU patients in the intervention hospitals were assumed to have received CHG bathing. DICON hospitals that reported complete data about the use of CHG bathing and surveillance data between January 2008 and December 2013 were included in the study.

This study was approved by the Duke University Institutional Review Board.

\section{Infections and Variables of Interest}

The study outcomes included 13 HAIs arising in the ICU in 3 major categories: MRSA infections, VRE infections, and non-species-specific infections. We analyzed HAIs due to MRSA and VRE by looking at 5 categories: central-line-associated bloodstream infection (CLABSI); ventilator-associated pneumonia (VAP); and catheter-associated urinary tract infection (CAUTI) due to MRSA or VRE; other HAIs that were not CLABSI, VAP, or CAUTI; and all HAIs due to MRSA or VRE. For non-species-specific (ie, "all") HAIs arising in the ICU, we examined primary bloodstream infections (BSIs), CLABSIs, VAP, and CAUTIs.

Additional covariates included whether or not the hospital was performing active MRSA surveillance and a proxy for hospital patient mix estimated using the ratio of ICU patient days to total patient days. ${ }^{24}$

\section{Statistical Analysis}

The effect of bathing with CHG was estimated using a multivariate piecewise Poisson mixed-effects model, also known as an interrupted time series model. We estimated the incidence rate ratio (IRR) and 95\% confidence interval (CI) for the effect of using CHG bathing compared to baseline, as well as the linear trends in incidence over time both before and after implementation of CHG bathing. Control hospitals were used as concurrent controls in the analysis. Each hospital's time series was calculated in months, centered on the date of the initiation of CHG bathing or the last time point in their series (for control hospitals). This allowed control hospitals to contribute to estimates of secular trends in infection rates and confounding variables and increased the precision of estimates prior to CHG bathing implementation. The effect of each random variable was estimated for the entire study period but was allowed to vary randomly within each hospital to account for both within- and between-hospital variability. The effect of initiating CHG bathing was modeled as a fixed effect that was not allowed to vary between hospitals; the time trends before and after CHG bathing was implemented were modeled as random effects. Higherorder terms for time trends were considered using nonlinear modeling, and the resulting Akaike Information Criterion were used to determine the ideal shape for time trends (ie, linear, quadratic, etc.). The 2 potential confounding variables, patient mix for each hospital and whether or not the hospital was engaged in active MRSA screening, were evaluated for inclusion in the multivariate model for each outcome using a 10\% change-in-estimate approach. 


\section{Results}

The study included 1,932 monthly observations of pro-spectively collected surveillance data from 33 hospitals, 17 (52\%) of which implemented CHG bathing during the 6-year study period. Hospitals that initiated CHG bathing protocols during the study period contributed 297,126 ICU patient days prior to implementing CHG bathing and 247,612 ICU patient days after implementing CHG bathing, for a total of 544,738 ICU patient days. Hospitals that implemented CHG bathing contributed a median of 33 months of surveillance data per hospital after implementation of CHG bathing (interquartile range, 6-51 months). Hospitals that did not use CHG bathing contributed 304,893 ICU patient days. Active MRSA screening was performed by 15 hospitals (88\%) that implemented CHG bathing during the study period and by 8 hospitals (50\%) that did not use CHG bathing.

\section{Baseline Analysis: Comparison of Aggregate Rates}

Hospitals that instituted protocols for CHG bathing had significantly higher overall incidence rates of total MRSA infections, total VRE infections, and all non-species-specific ICU infections compared with hospitals that did not implement CHG bathing (Table 1). Hospitals that implemented CHG bathing during the study period had significantly higher initial incidence rates of all combined MRSA infections and all combined VRE infections (Table 2). The incidence rates of non-species-specific ICU primary BSI significantly increased after implementation of CHG bathing among hospitals that started using $\mathrm{CHG}$ during the study period.

We reviewed species-specific data for 303 ICU CLABSIs before and after the implementation of CHG bathing (Table 3). Gram-positive bacteria were less commonly identified after initiation of CHG bathing, whereas Gram-negative bacteria and yeast species were more commonly identified after initiation of CHG bathing; however, these trends were not significant.

\section{Interrupted Time Series Analysis: Rate Trends}

CHG bathing was associated with significant downward trends in incidence rates of ICU CLABSIs and ICU primary BSIs (Table 4). The incidence rate of ICU CLABSIs had a significant downward trend prior to implementation of CHG bathing, but the postimplementation trend was steeper (Figure A). Hospitals that implemented CHG bathing during the study period had a $1 \%$ decrease in ICU CLABSIs each month before implementation and a $4 \%$ decrease in ICU CLABSIs each month after implementation. ICU primary BSIs did not display a downward trend prior to CHG implementation, but there was a 4\% decrease in BSIs each month after CHG bathing was implemented (Figure B).

CHG bathing was similarly associated with significant, downward trends in incidence rates of VRE CLABSIs and all combined VRE infections (Table 4). The incidence rate of VRE CLABSIs exhibited a significant downward trend prior to CHG bathing implementation and a steeper post-implementation trend. Hospitals that implemented CHG bathing during the study period had a 1\% decrease in VRE CLABSIs each month before CHG bathing implementation and a 3\% decrease in VRE CLABSIs each month after CHG bathing 
implementation. All combined VRE infections did not display a downward trend prior to CHG implementation, but there was a 4\% decrease in VRE infections each month after CHG bathing was implemented.

The incidence rate of VRE CAUTIs decreased significantly prior to implementation of CHG bathing, but this trend was not seen after the implementation of CHG bathing. The incidence rate of non-species-specific ICU CAUTIs did not change before or after implementation of CHG bathing.

MRSA infection incidence rates (CLABSI, VAP, other, or all combined MRSA infections) did not significantly change prior to or following implementation of CHG bathing. Issues with the convergence of the statistical model prevented examination of MRSA CAUTI infections. The incidence rate of non-CLABSI, non-CAUTI VRE infections also was not affected by CHG bathing implementation. The rate of non-species-specific ICU VAPs decreased significantly prior to CHG bathing implementation, but this trend was not seen after $\mathrm{CHG}$ bathing was implemented.

Patient mix for each hospital and whether or not the hospital was engaged in active MRSA screening did not impact any estimates and thus were not included in the final models. Notably, for a number of the HAI incidence trends, a sharp increase or decrease in HAI incidence rate occurred during the first month after CHG bathing was implemented. This is likely the result of the pre-intervention time series, in which the data from all hospitals was used, whereas the post-intervention time series, by its very nature, only leveraged data from those hospitals that implemented CHG bathing. The immediate changes in HAI incidence rates are more likely statistical artifacts from the different pre-existing infection rates between control hospitals and those that implemented CHG bathing, rather than an outcome of the CHG bathing intervention itself.

\section{Discussion}

Incidence rates of several important HAIs, including ICU CLABSIs, ICU primary BSIs, and VRE CLABSIs, declined after the implementation of CHG bathing in ICUs. Hospitals that implemented CHG bathing achieved a 59\% decrease in ICU CLABSIs, a 36\% decrease in ICU primary BSIs, and a 33\% decrease in VRE CLABSIs after using CHG bathing for 12 months. Our findings largely support the results from previously published multicenter trials. 17,19,20 However, our study was performed in multiple community hospitals in a real-world setting; thus, our study is the first to support the use of CHG bathing to decrease HAIs in ICU patients in a type 3 translational research setting.

Overall, our results support the findings of Climo et al, ${ }^{17}$ who conducted a multicenter, cluster-randomized, nonblinded crossover study in which CHG bathing led to a significant decreases in primary BSIs, CLABSIs, and VRE infections, but not to a decrease in MRSA infections. Huang et $\mathrm{al}^{19}$ and Milstone et $\mathrm{al}^{20}$ also found that $\mathrm{CHG}$ bathing led to a lower incidence of BSIs in their multicenter, cluster-randomized trials. Our findings contrast with those of Noto et al, ${ }^{18}$ whose single-center, cluster-randomized, crossover study found that daily bathing with CHG did not reduce the incidence of CLABSIs. 
Notably, implementation of CHG bathing did not significantly affect the incidence of MRSA CLABSIs, MRSA VAPs, or other MRSA infections. Hospitals that implemented CHG bathing exhibited a trend toward fewer MRSA CLABSIs; MRSA non-CLABSI, non-VAP infections; and all MRSA infections. It is possible that too few MRSA infections occurred at the study hospitals to realize a significant difference. However, other studies have also reported that CHG bathing does not impact MRSA infection rates. ${ }^{17,25}$ This finding further underscores the importance of using interrupted time series methods over strict before-andafter analyses. Our baseline analysis comparing aggregate rates of the different types of MRSA infections suggested that the rates were lower after implementing CHG bathing. In contrast, our controlled, multivariate, interrupted time series analysis did not support this conclusion. In other words, hospitals that evaluate the impact of interventions like CHG bathing using a single-center, quasi-experimental approach may lead to incorrect conclusions.

Lower rates of CLABSI due to Gram-positive cocci accounted for most of the observed decline in CLABSI rates in ICUs that implemented CHG bathing. The most common pathogen causing CLABSIs in hospitals prior to implementing CHG bathing were coagulase-negative Staphylococci(CoNS), whereas yeast species and Gram-negative bacteria became the most common causes of CLABSI after CHG bathing implementation. Other studies also saw a decrease in CoNS CLABSIs after the implementation of CHG bathing. ${ }^{17,19,20}$ While CLABSIs due to CoNS may often be contaminants, we believe that they are still treated as pathogens by clinicians. Thus, we conclude that decreasing the rate of CLABSIs caused by CoNS remains an important outcome for ICU patients.

CHG bathing to prevent CLABSIs is recommended for all ICU patients, ${ }^{26}$ but it is unclear whether hospitals with low baseline infection rates gain additional benefit from $\mathrm{CHG}$ bathing. In our study, hospitals that chose not to implement CHG bathing had significantly lower incidence rates of CAUTIs, CLABSIs, primary BSIs, all combined MRSA infections, and all combined VRE infections than did hospitals that implemented CHG bathing. Hospitals that did not implement CHG bathing also had a lower number of ICU patient days. One interpretation of these findings is that some hospitals, perhaps those with lower ICU utilization, may not need to use CHG bathing in their ICUs. It is possible that there is a threshold of MDRO colonization pressure and patient volume above which hospitals benefit from CHG bathing. Our study was not designed to evaluate this question, and further studies are warranted.

Our study has several limitations. First, our standard, prospective surveillance approach only included the specific outcomes described above. There may be additional important outcomes and confounders that we did not measure. The study population consists of hospitals that chose to participate in an infection control network, which may have caused selection bias. Additionally, we were not able to assess adherence to CHG bathing protocols or $\mathrm{CHG}$ bathing techniques. However, the practical use of $\mathrm{CHG}$ bathing will not be a controlled process, and thus we believe that our findings are representative of real-world use of CHG bathing. Our large cohort of community hospitals and the large amount of data that we accumulated over the study period further strengthens the applicability of our results. 
In conclusion, our interrupted time series analysis suggests that hospitals that implemented CHG bathing over the study period achieved decreases in ICU CLABSIs, ICU primary BSIs, and VRE CLABSIs, but CHG bathing did not affect MRSA infection rates. Thus, our findings further support the use of daily CHG bathing of ICU patients, particularly in hospitals with high incidence rates of ICU CLABSIs, ICU primary BSIs, and VRE CLABSIs.

\section{Acknowledgments}

Financial support: Dr. Anderson was supported by NIAID/NIH (grant no. K23AI095357).

\section{References}

1. Magill S, Edwards J, Bramberg W, et al. Multistate point-prevalence survey of health careassociated infections. N Engl J Med. 2014; 370:1198-1208. [PubMed: 24670166]

2. Zimlichman E, Henderson D, Tamir O, et al. Health care-associated infections: a meta-analysis of costs and financial impact on the US health care system. JAMA Intern Med. 2013; 173:2039-2046. [PubMed: 23999949]

3. Cosgrove SE, Sakoulas G, Perencevich EN, Schwaber MJ, Karchmer AW, Carmeli Y. Comparison of mortality associated with methicillin-resistant and methicillin-susceptible Staphylococcus aureus bacteremia: a meta-analysis. Clin Infect Dis. 2003; 36:53-59. [PubMed: 12491202]

4. Blot SI, Vandewoude KH, Hoste EA, Colardyn FA. Outcome and attributable mortality in critically ill patients with bacteremia involving methicillin-susceptible and methicillin-resistant Staphylococcus aureus. Arch Intern Med. 2002; 162:2229-2235. [PubMed: 12390067]

5. Abramson MA, Sexton DJ. Nosocomial methicillin-resistant and methicillin-susceptible Staphylococcus aureus primary bacteremia: at what costs? Infect Control Hosp Epidemiol. 1999; 20:408-411. [PubMed: 10395142]

6. Cosgrove SE, Qi Y, Kaye KS, Harbarth S, Karchmer AW, Carmeli Y. The impact of methicillin resistance in Staphylococcus aureus bacteremia on patient outcomes: mortality, length of stay, and hospital charges. Infect Control Hosp Epidemiol. 2005; 26:166-174. [PubMed: 15756888]

7. DiazGranados CA, Zimmer SM, Klein M, Jernigan JA. Comparison of mortality associated with vancomycin-resistant and vancomycin-susceptible enterococcal bloodstream infections: a metaanalysis. Clin Infect Dis. 2005; 41:327-333. [PubMed: 16007529]

8. Popovich KJ, Lyles R, Hayes R, et al. Relationship between chlorhexidine gluconate skin concentration and microbial density on the skin of critically ill patients bathed daily with chlorhexidine gluconate. Infect Control Hosp Epidemiol. 2012; 33:889-896. [PubMed: 22869262]

9. Popovich KJ, Hota B, Hayes R, Weinstein RA, Hayden MK. Effectiveness of routine patient cleansing with chlorhexidine gluconate for infection prevention in the medical intensive care unit. Infect Control Hosp Epidemiol. 2009; 30:959-963. [PubMed: 19712033]

10. Vernon MO, Hayden MK, Trick WE, et al. Chlorhexidine gluconate to cleanse patients in a medical intensive care unit: the effectiveness of source control to reduce the bioburden of vancomycin-resistant enterococci. Arch Intern Med. 2006; 166:306-312. [PubMed: 16476870]

11. Darouiche RO, Wall MJ Jr, Itani KM, et al. Chlorhexidine-alcohol versus povidone-iodine for surgical-site antisepsis. N Engl J Med. 2010; 362:18-26. [PubMed: 20054046]

12. Armellino D, Woltmann J, Parmentier D, et al. Modifying the risk: once-a-day bathing "at risk" patients in the intensive care unit with chlorhexidine gluconate. Am J Infect Control. 2014; 42:571-573. [PubMed: 24773800]

13. Cassir N, Thomas G, Hraiech S, et al. Chlorhexidine daily bathing: impact on health careassociated infections caused by Gram-negative bacteria. Am J Infect Control. 2015; 43:640-643. [PubMed: 25798778]

14. Popovich KJ, Hota B, Hayes R, Weinstein RA, Hayden MK. Daily skin cleansing with chlorhexidine did not reduce the rate of central-line associated bloodstream infection in a surgical intensive care unit. Insensive Care Med. 2010; 36:854-858. 
15. Viray MA, Morley JC, Coopersith CM, Kollef MH, Fraser VJ, Warren DK. Daily bathing with chlorhexidine-based soap and the prevention of Staphylococcus aureus transmission and infection. Infect Control Hosp Epidemiol. 2014; 35:243-250. [PubMed: 24521588]

16. Climo MW, Sepkowitz KA, Zuccotti G, et al. The effect of daily bathing with chlorhexidine on the acquisition of methicillin-resistant Staphylococcus aureus, vancomycin-resistant Entero-coccus, and healthcare-associated bloodstream infections: results of a quasi-experimental multicenter trial. Crit Care Med. 2009; 37:1858-1865. [PubMed: 19384220]

17. Climo MW, Yokoe DS, Warren DK, et al. Effect of daily chlorhexidine bathing on hospitalacquired infection. N Engl J Med. 2013; 368:533-542. [PubMed: 23388005]

18. Noto MJ, Domenico HJ, Byrne DW, et al. Chlorhexidine bathing and health care-associated infections: a randomized clinical trial. JAMA. 2015; 313:369-378. [PubMed: 25602496]

19. Huang SS, Septimus E, Kleinman K, et al. Targeted versus universal decolonization to prevent ICU infection. N Engl J Med. 2013; 368:2255-2265. [PubMed: 23718152]

20. Milstone AM, Elward A, Song X, et al. Daily chlorhexidine bathing to reduce bacteraemia in critically ill children: a multi-center, cluster-randomised, crossover trial. Lancet. 2013; 381:1099_ 1106. [PubMed: 23363666]

21. Chen W, Li S, Li L, Wu X, Zhang W. Effects of daily bathing with chlorhexidine and acquired infection of methicillin-resistant Staphylococcus aureus and vancomycin-resistant Enterococcus: a meta-analysis. J Thorac Dis. 2013; 5:518-524. [PubMed: 23991311]

22. O'Horo JC, Silva GL, Munoz-Price LS, Safdar N. The efficacy of daily bathing with chlorhexidine for reducing healthcare-associated bloodstream infections: a meta-analysis. Infect Control Hosp Epidemiol. 2012; 33:257-267. [PubMed: 22314063]

23. Anderson DJ, Miller BA, Chen LF, et al. The network approach for prevention of healthcareassociated infections: long-term effect of participation in the Duke Infection Control Outreach Network. Infect Control Hosp Epidemiol. 2011; 32:315-322. [PubMed: 21460482]

24. Moehring RW, Lofgren ET, Anderson DJ. Impact of change to molecular testing for Clostridium difficile infection on healthcare facility-associated incedence rates. Infect Control Hosp Epidemiol. 2013; 34:1055-1061. [PubMed: 24018922]

25. Karki S, Cheng AC. Impact of non-rinse skin cleansing with chlorhexidine gluconate on prevention of healthcare-associated infections and colonization with multi-resistant organisms: a systematic review. J Hosp Infect. 2012; 82:71-84. [PubMed: 22889522]

26. Marschall J, Mermel LA, Fakih M, et al. Strategies to prevent central line-associated bloodstream infections in acute care hospitals: 2014 update. Infect Control Hosp Epidemiol. 2014; 35:753-771. [PubMed: 24915204] 

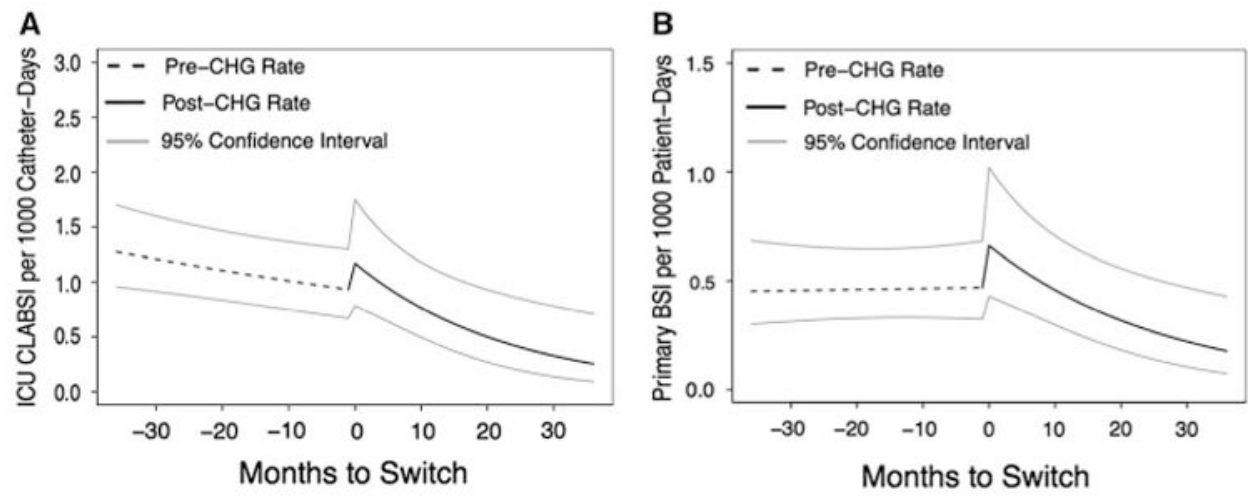

Figure 1.

Incidence rates of hospital-acquired infections in intensive care unit (ICU) patients before and after implementation of chlorhexidine gluconate bathing at 17 community hospitals are compared against incidence rates of hospital-acquired infections in ICU patients in 16 community hospitals that did not use chlorhexidine gluconate bathing for the following infections: (A) central-line associated bloodstream infection (CLABSI); (B) primary bloodstream infection (BSI). 


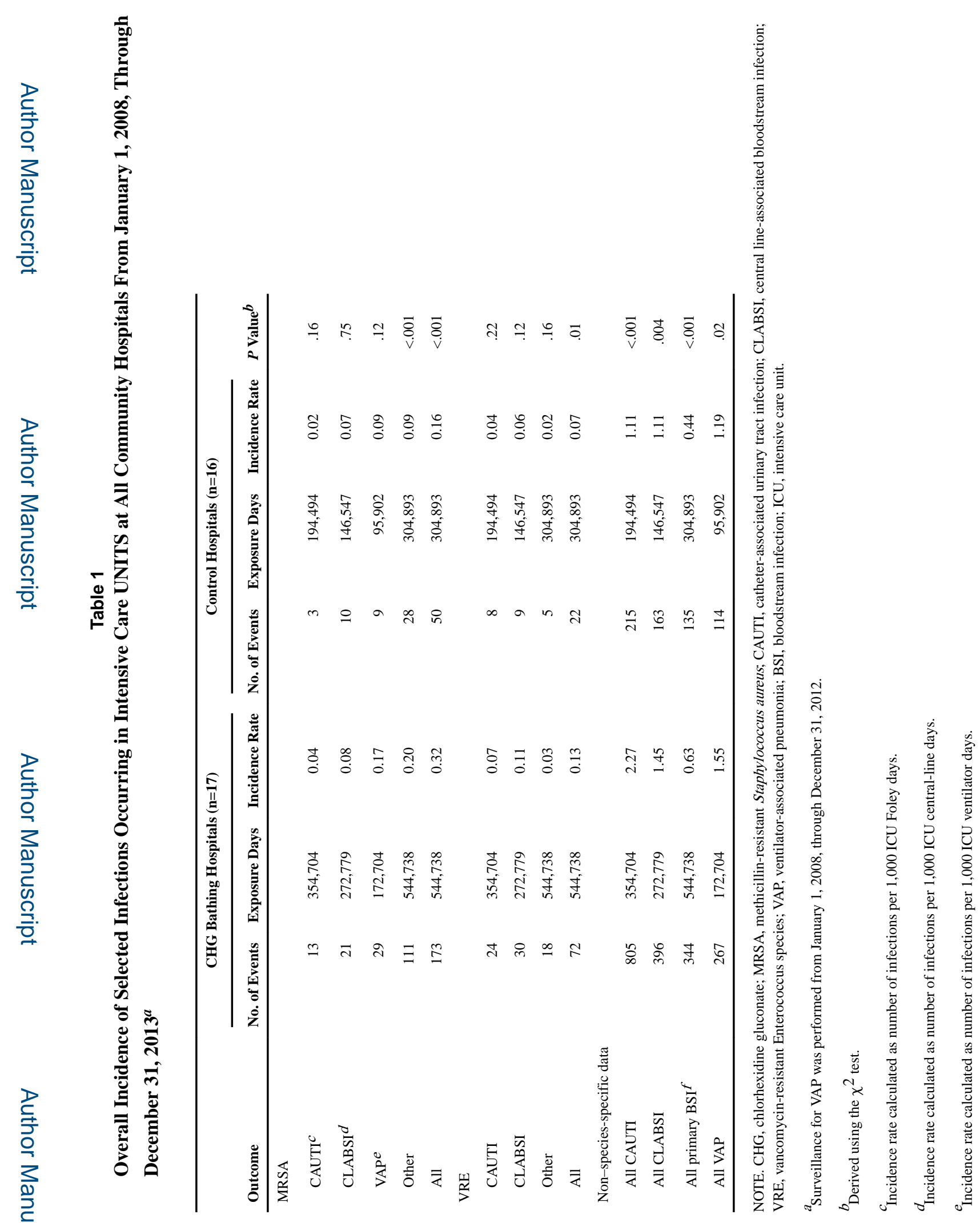

Infect Control Hosp Epidemiol. Author manuscript; available in PMC 2018 April 11. 
Dicks et al.

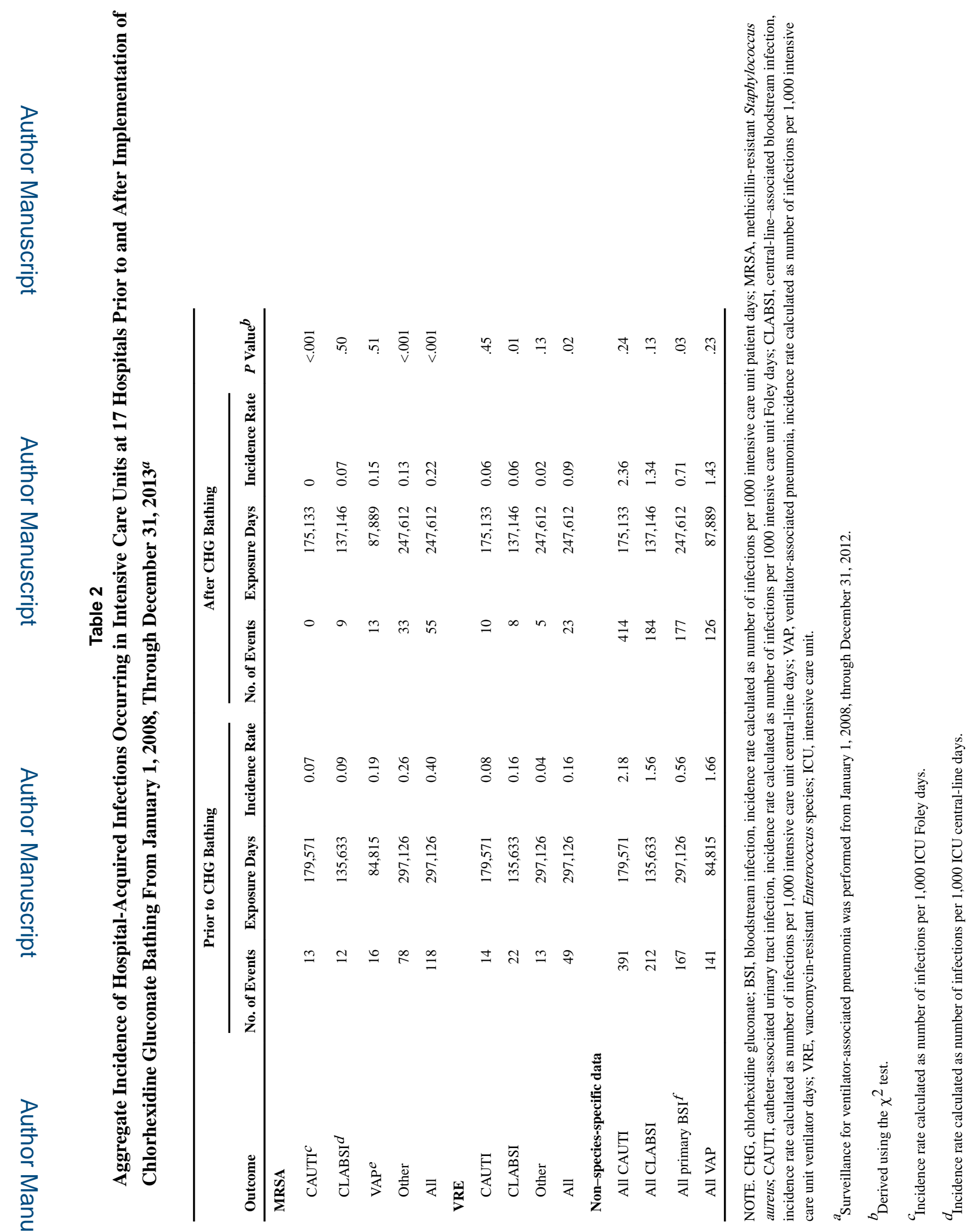

Infect Control Hosp Epidemiol. Author manuscript; available in PMC 2018 April 11. 


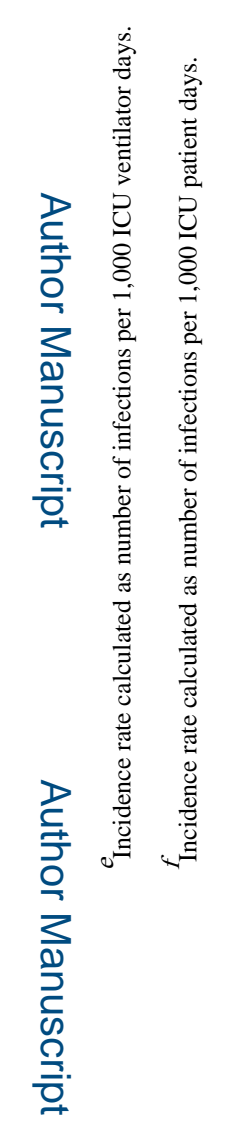

로을

로을

Infect Control Hosp Epidemiol. Author manuscript; available in PMC 2018 April 11. 
Table 3

Microorganisms Isolated From CLABSIs Occurring in ICUs at 17 Community Hospitals Prior to and After Implementing Chlorhexidine Gluconate Bathing

\begin{tabular}{|c|c|c|c|}
\hline Organism & $\begin{array}{l}\text { Prior to CHG Bathing Implementation, } \\
\text { No. }(\%)(\mathbf{N}=151)\end{array}$ & $\begin{array}{l}\text { After CHG Bathing Implementation, } \\
\text { No. }(\%)(\mathbf{N}=152)\end{array}$ & $P$ Value ${ }^{a}$ \\
\hline Gram-positive bacteria & $91(60)$ & $74(49)$ & .16 \\
\hline Coagulase-negative Staphylococci & $38(25)$ & $31(20)$ & .37 \\
\hline Enterococcus spp. & $36(24)$ & $20(13)$ & .03 \\
\hline MRSA & $12(8)$ & $9(6)$ & .50 \\
\hline MRSA & $4(3)$ & $9(6)$ & .17 \\
\hline Other & $1(1)$ & $5(3)$ & .11 \\
\hline Gram-negative bacteria & $30(20)$ & $35(23)$ & .57 \\
\hline Yeast & $28(19)$ & $41(27)$ & .13 \\
\hline Other & $2(1)$ & $2(1)$ & .99 \\
\hline Total & 151 & 152 & \\
\hline
\end{tabular}

NOTE. CLABSI, central-line-associated bloodstream infection; ICU, intensive care unit; CHG, chlorhexidine gluconate; MRSA, methicillinsensitive Staphylococcus aureus.

${ }^{a}$ Based on crude incidence rate ratios. 
Table 4

Adjusted Incidence Rate Ratios and Linear Trends in Incidence Over Time Before and After Chlorhexidine Gluconate Bathing Implementation ${ }^{a}$

\begin{tabular}{|c|c|c|c|}
\hline Outcome & $\begin{array}{l}\text { CHG Bathing IRR Ratio (95\% } \\
\text { CI) } b\end{array}$ & $\begin{array}{l}\text { Pre-CHG Bathing Incidence Rate } \\
\qquad(95 \% \mathrm{CI})^{c}\end{array}$ & $\begin{array}{c}\text { Post-CHG Bathing Incidence } \\
\text { Rate }(95 \% \text { CI })^{c}\end{array}$ \\
\hline \multicolumn{4}{|l|}{ MRSA } \\
\hline CLABSI $^{d}$ & $2.92(0.80-10.64)$ & $0.96(0.98-1.01)$ & $0.95(0.90-1.01)$ \\
\hline $\mathrm{VAP}^{e}$ & $1.71(0.50-5.83)$ & $0.97(0.94-1.01)$ & $0.97(0.93-1.01)$ \\
\hline Other & $0.95(0.48-1.89)$ & $1.00(0.98-1.02)$ & $0.99(0.96-1.01)$ \\
\hline All & $1.16(0.68-1.98)$ & $0.99(0.98-1.00)$ & $0.98(0.96-1.00)$ \\
\hline \multicolumn{4}{|l|}{ VRE } \\
\hline $\mathrm{CAUTI}^{f}$ & $2.10(0.57-7.81)$ & $0.97(0.95-1.00)$ & $0.97(0.93-1.02)$ \\
\hline CLABSI & $0.81(0.81-0.82)$ & $0.99(0.99-1.00)$ & $0.97(0.97-0.98)$ \\
\hline Other & $1.44(0.34-6.11)$ & $1.03(0.99-1.08)$ & $0.90(0.80-1.01)$ \\
\hline All & $1.19(0.54-2.65)$ & $1.00(0.98-1.02)$ & $0.96(0.93-1.00)$ \\
\hline \multicolumn{4}{|c|}{ Non-species-specific data } \\
\hline All CAUTI & $0.99(0.77-1.28)$ & $1.00(1.00-1.01)$ & $1.00(0.98-1.01)$ \\
\hline All CLABSI & $1.27(0.91-1.76)$ & $0.99(0.98-1.00)$ & $0.96(0.93-0.99)$ \\
\hline All primary BSI $g$ & $1.41(0.98-2.02)$ & $1.00(0.99-1.01)$ & $0.96(0.94-0.99)$ \\
\hline All VAP & $1.91(1.25-2.94)$ & $0.98(0.97-1.00)$ & $0.99(0.97-1.00)$ \\
\hline
\end{tabular}

NOTE. CHG, chlorhexidine gluconate; IRR, implementation incidence rate; CI, confidence interval; MRSA, methicillin-resistant Staphylococcus aureus; CLABSI, central-line-associated bloodstream infection; VAP, ventilator-associated pneumonia; VRE, vancomycin-resistant Enterococcus species; CAUTI, catheter-associated urinary tract infection; BSI, bloodstream infection; ICU, intensive care unit.

a Estimates obtained using a multivariate piecewise Poisson mixed-effects model. Surveillance for VAP was performed from January 1, 2008, through December 31, 2012. MRSA CAUTIs could not be modeled.

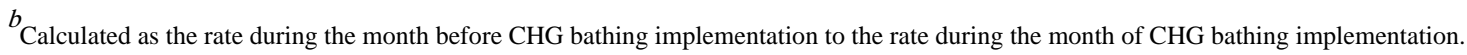

$c$ Monthly change in rate of infection

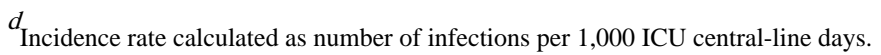

Incidence rate calculated as number of infections per 1,000 ICU ventilator days.

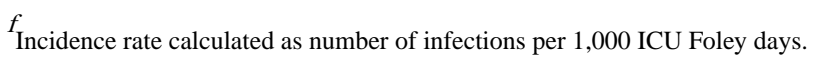

$g_{\text {Incidence rate calculated as number of infections per 1,000 ICU patient days. }}$ 